\title{
Measurements of Functional Response of Nano-objects using Advanced Electron Microscopy
}

Y. Zhu, T. Beetz, L. Wu, R. Klie, L. Huang, J. W. Lau* , M. A. Schofield, V. V. Volkov, M. Beleggia, and M. Malac**

Center for Functional Nanomaterials, Brookhaven National Laboratory, Upton, NY 11973

* Current address: National Institute of Standards and Technology, Gaithersburg, MD 20899

** National Institute for Nanotechnology, Edmonton, T6G 2V4, Canada

One of the grand challenges in nanoscience and nanotechnology is the ability not only to characterize the local structure, but also to measure the functional response of individual objects at the nanometer scale. To meet this challenge, we have acquired several state-of-the-art, customdesigned instruments, including a low-aberration field-free microscope dedicated for magnetic imaging, and an aberration-corrected energy-filtered microscope for high-resolution STEM/TEM. Additionally, we have developed specialized sample stages ourselves [1], as well as in collaboration with manufacturers, for in-situ magnetization and current-voltage application to nano-objects, all the while monitoring their local atomic, electronic and magnetic structure.

To tailor nano-objects for study, we have extended TEM beam-writing methods to produce nanometer dots, and employed lift-off lithography techniques to fabricate micron and submicron elements with different shapes, sizes and array geometry (Fig.1(a)). Recently, we have used selfassembled polystyrene spheres as a mask to spin-coat magnetic films (Fig.1(b,c)). By varying the deposition and annealing conditions we are able to produce circular, triangular, and toroidal elements. Quantitative microscopy methods to determine the local magnetization distribution, using electron holography and Lorentz phase microscopy, were also developed to understand the magnetic properties and shape effect of individual elements, and their interaction with neighboring elements. Some examples are shown in Fig.1(d-e).

We also have been working on nanotubes and nanowires. Our carbon nanotube (CNT) study was in collaboration with L. Brus' group at Columbia University [2]. The electrical properties of these individual CNTs were measured at Columbia using Rayleigh spectroscopy, while the same tubes were then characterized at Brookhaven to determine their microstructure and chiral vector in order to establish the structure-property relationship of individual tubes. Fig.2(a) is a diffraction pattern from a semiconducting single-wall CNT (chiral index: 27,11) superimposed on a simulated one. The hexagonal patterns from the curved top and bottom graphine sheets are also indicated. In Fig.2(d), a custom-made holder developed by Nanofactroy Inc. (via Gatan) is shown, where the piezo-controlled STM tip allows us to measure I-V curves of individual well-characterized nanotubes. Changing the probe of this specialized stage to a magnetic tip, we can apply a local magnetic field and field gradient to individual magnetic elements, including elements of a frustrated array, in order to shed light on the interaction between spins and electrons, as well as on the reversal mechanism of nanoscale magnetic building blocks [3].

\section{References:}

[1] J.W. Lau, PhD Thesis, Columbia University, NY, 2005.

[2] M.Y. Sfeir, et al., under review for Science.

[3] This work was supported by U.S. DOE, under contract No. DE-AC02-98CH10886. 


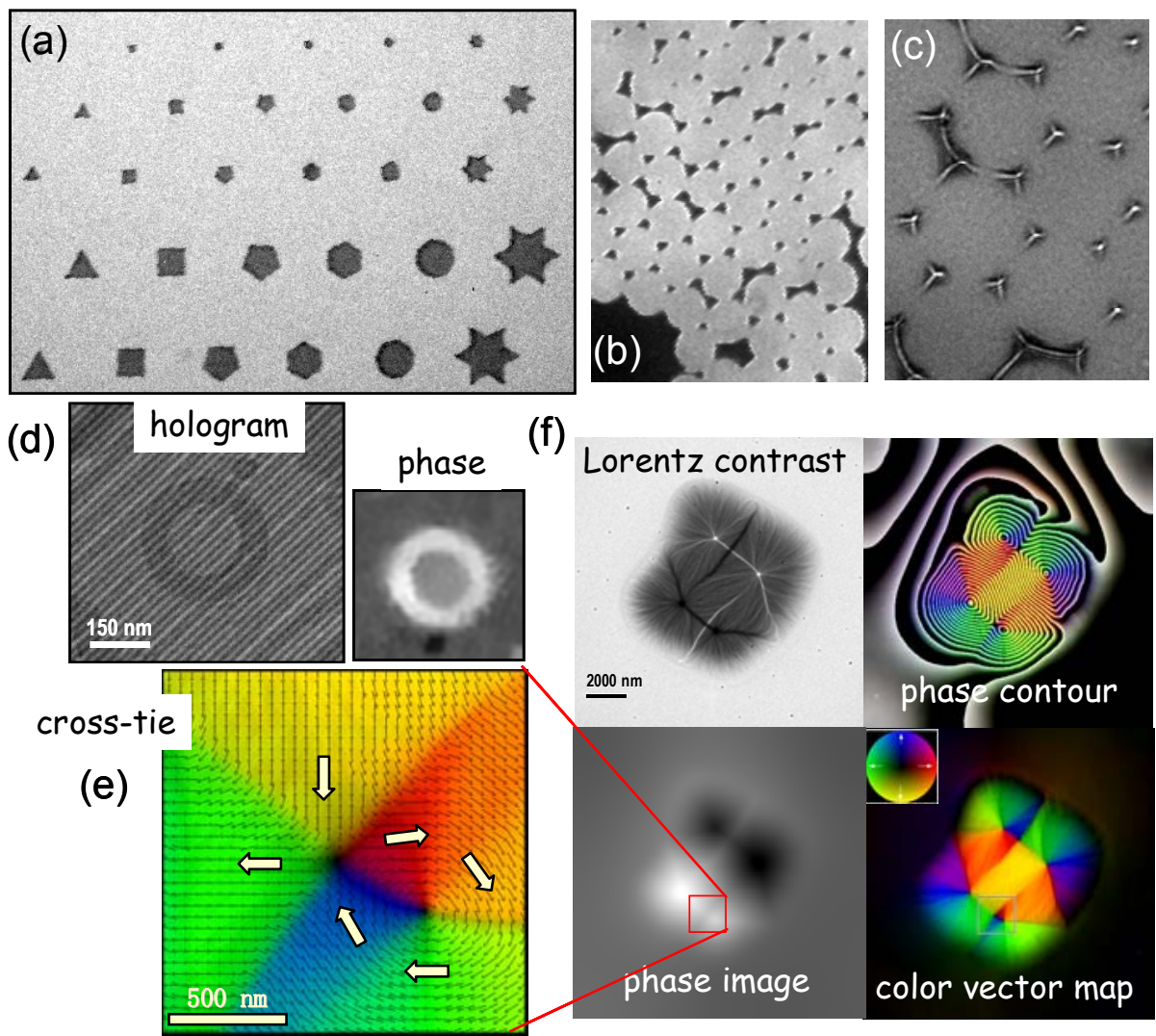

FIG. 1. (a): Lithographically patterned magnetic elements (Permalloy). The top-row elements are $\sim 100 \mathrm{~nm}$. (b) Ni islands fabricated via e-beam deposition after removal of the mask of $290 \mathrm{~nm}$ polystyrene-spheres. (c) Lorentz image of the $\mathrm{Ni}$ islands from (b) showing phase contrast with the local electrostatic and magnetic information encoded. (d) Experimental hologram and reconstructed phase image of a Permalloy ring. (f) Phase retrieval of a magnetic element using Lorentz phase microscopy. The magnetic vector map from a local area in (f) is shown in (e). A color legend for the vector amplitude and direction is also included.
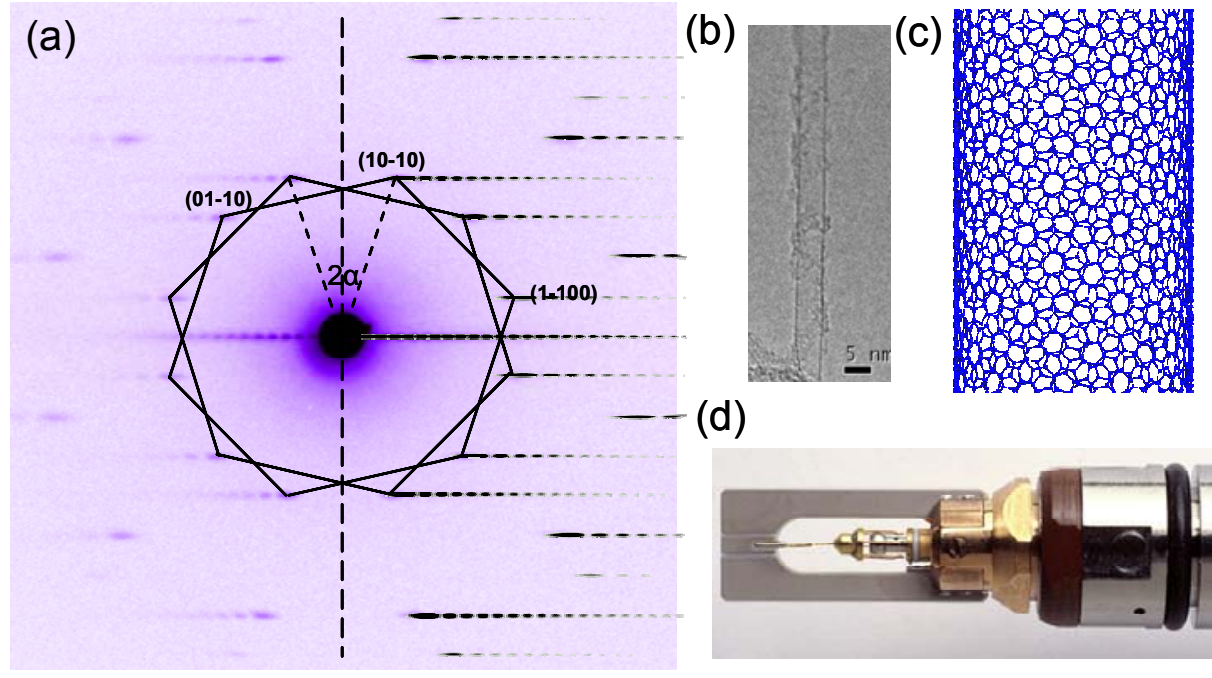

(d)

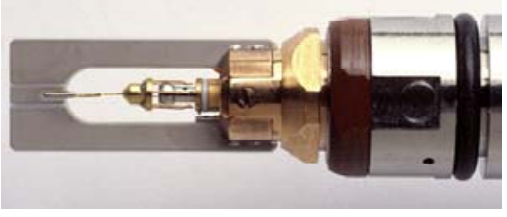

FIG. 2. (a) Experimental diffraction from a single-wall nanotube (b), superimposed with a simulated pattern at right. The measured chiral index is $(27,11)$ and chiral angle $16.34^{\circ}$ (which is not $\alpha$ ); (c) The structural model. (d) The STM tip on a TEM holder custom-designed by Nanofacory Inc. It allows us to manipulate individual nanoobjects inside a microscope and to measure their physical properties and functional response. 